\title{
Seven fractions to deliver partial breast irradiation: the toxicity is Low
}

\author{
Marco Trovo ${ }^{1}$, Michele Avanzo ${ }^{2^{*}} \mathbb{D}$, Lorenzo Vinante ${ }^{1}$, Carlo Furlan ${ }^{1}$, Francesco Fiorica ${ }^{3}$, Tiziana Perin ${ }^{4}$, \\ Loredana Militello ${ }^{5}$, Simon Spazzapan ${ }^{5}$, Massimiliano Berretta ${ }^{5}$, Rajesh Jena ${ }^{6}$, Joseph Stancanello ${ }^{7}$, Erica Piccoli ${ }^{8}$, \\ Mario Mileto ${ }^{8}$, Elvia Micheli ${ }^{9}$, Mario Roncadin ${ }^{1}$ and Samuele Massarut ${ }^{8}$
}

\begin{abstract}
Purpose: To assess toxicity and clinical outcome, in breast cancer patients treated with external beam partial breast irradiation (PBI) consisting of 35 Gy in 7 daily fractions (5 Gy/fraction).

Materials and Methods: Patients affected by early-stage breast cancer were enrolled in this phase II trial. Patients had to be 60 years old or over and treated with breast conservative surgery for early stage invasive carcinoma.

Results: Seventy-three patients were analyzed. Median follow-up was 40 months. The proposed schedule was well tolerated. No Grade 3 toxicity was documented. Late toxicity was assessable for all the treated patients. Two patients (2.7\%) developed Grade 2 pain 6 months after PBI. Four patients (5\%) developed asymptomatic fat necrosis. Grade 2 fibrosis was observed in 5 patients (6.7\%). No correlation was found between early and late toxicity and the type of adjuvant systemic therapy (no therapy vs. hormonal therapy vs. chemotherapy). No statistical correlation between dosimetric parameters and toxicity was found. Patients who developed Grade 2 radiation fibrosis had not higher radiation volumes to the untreated normal breast than those without fibrosis. Cosmesis was judged good/excellent in the majority of the cases (93\%). One patient relapsed locally, and one developed distant metastases, corresponding to a 5-year local control and distant metastases-free survival of $98 \%$ and $96.7 \%$, respectively.
\end{abstract}

Conclusions: 35 Gy in 7 daily fractions is an effective and well-tolerated regimen to deliver PBI.

Keywords: Partial breast irradiation, Breast cancer, Toxicity, Fractionation

\section{Introduction}

Although whole breast irradiation remains the standard of care after conservative surgery for early stage breast cancer, partial breast irradiation (PBI), namely the irradiation of only the breast tissue surrounding the lumpectomy cavity, is emerging. The recent publication of the American Society for Radiation Oncology (ASTRO) consensus statement gave a further stimulus to the spread of PBI in clinical practice [1].

The most used fractionation scheme is that proposed by Vicini at al., who explored in a phase II trial the safety and efficacy of 38.5 Gy at 3.85 Gy/fraction delivered 2 fractions per day with three dimensional conformal external beam radiotherapy (3D-CRT) [2]. This fractionation

\footnotetext{
* Correspondence: mavanzo@cro.it

²Division of Medical Physics, Centro di Riferimento Oncologico IRCSS, 33081

Aviano, Italy

Full list of author information is available at the end of the article
}

was then adopted also by the Radiation Therapy Oncology Group (RTOG): the RTOG 0319 study documented that delivering 38.5 Gy in 5 days, 2 fractions per day, is safe, and the toxicity profile is low [3].

Due to the fact that some studies reported poor cosmetic and toxicity results using the above-mentioned radiotherapy schedule $[4,5]$, we treated our patients with PBI using a once daily fractionation scheme consisting of $40 \mathrm{~Gy}$ in 10 fractions, and we documented an excellent toxicity profile [6]. We therefore decided to continue to accelerate the radiation delivery, according to previously published radiobiological models $[7,8]$.

The purpose of the present study is to assess prospectively the efficacy and toxicity in breast cancer patients treated who underwent PBI with a schedule consisting of 35 Gy in 7 daily fractions (5 Gy per fraction). 


\section{Materials and Methods Patients}

Between July 2011 and December 2013 patients affected by early stage breast cancer, who had breast conservative surgery were enrolled in this phase II prospective trial. Both the approval from our institutional review board and written informed consent from the patients were obtained.

To be included in this study patients had to be 60 years old or over and treated with breast conservative surgery for early stage (pT1-T2 pN0-N1a) invasive ductal (IDC) or lobular carcinoma (ILC). Patients were required to have negative surgical margins; re-excision was allowed in case of positive margins. Biopsy of sentinel lymph node was not required. Patients affected by ductal carcinoma in-situ were not included in this study. Adjuvant hormonal therapy or chemotherapy were allowed. In case of chemotherapy, radiation started one month after the completion of it.

\section{Treatment}

Radiation therapy consisted of 35 Gy delivered in 7 daily fractions, $5 \mathrm{~Gy} /$ fraction. At least three radio-opaque fiducial markers (surgical clips) were placed in the tumor bed at the time of surgery [9]. Treatment planning for radiation was performed by immobilizing patients in supine position with the Quest Breastboard (Q-Fix System). All patients underwent a complete free breathing computed tomography (CT) simulation to include all the organs at risk (OAR), according to the RTOG 0413 protocol [http://www.rtog.org/ members/protocols/0413/0413.pdf]. The CT simulation was performed not before two months following surgery.
The clinical target volume (CTV) consisted of the lumpectomy cavity, identified by the surgical clips, uniformly expanded by $10 \mathrm{~mm}$, limited to $5 \mathrm{~mm}$ from the skin surface and $5 \mathrm{~mm}$ from the lung-chest wall interface. The planning target volume (PTV) was calculated from the CTV using uniform three-dimensional expansion of $5 \mathrm{~mm}$. PTV for evaluation (PTV-EVAL) is the structure used for dosevolume histogram (DVH) constraints and analysis; it is limited to exclude the part outside the ipsilateral breast and the first $5 \mathrm{~mm}$ of tissue under the skin and excluding the PTV expansion beyond the posterior extent of breast tissue. Radiation therapy was delivered to the PTV using three dimensional conformal fields, adopting the "field within a field" technique (forward Intensity Modulated Radiation Therapy technique) to improve dose homogeneity within the PTV and dosimetric coverage, as previously described [10]. Dose calculation with tissue inhomogeneity correction was used. All treatments were developed using the Eclipse treatment planning system (Varian Medical Systems, Palo Alto, CA, USA), using multiple planar and non-coplanar 6-MV photon beams. Fig. 1 shows a typical dose distribution. After a treatment plan was approved, digitally reconstructed radiographs (DRRs) were constructed to show each fiducial marker. Radiotherapy treatment commenced 3 to 4 weeks after simulation. The treatment was delivered by a Trilogy linear accelerator equipped with a $\mathrm{kV}$ on-board imager system and a 120-leaves Millennium multi-leaf collimator (Varian Medical Systems).

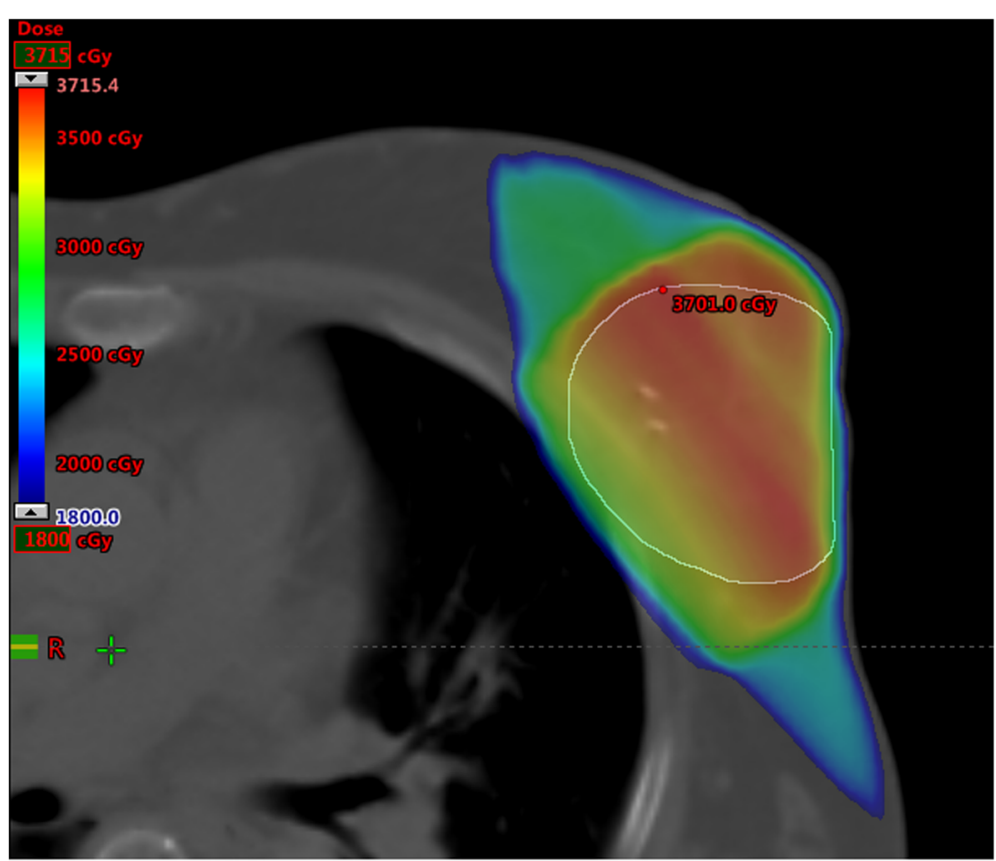

Fig. 1 Dose distribution for a partial breast irradiation plan (upper-outer quadrant of the left breast) 


\section{Follow-up and statistics}

Patients were seen at regular intervals to determine the presence of symptoms, and physicians evaluated toxicity by Common Toxicity Criteria of Adverse Events, version 3.0. Follow-up visits for the evaluation of toxicity occurred 1, 3, 6 and 12 months from the completion of PBI for the first year, and then once a year. Cosmesis was assessed using the Harvard scale. Dosimetric parameters from the subgroup with and without Grade 2 or greater radiation induced breast fibrosis were compared using a two-tailed Student's $t$ test; statistical significance was claimed for $p<0.05$.

\section{Results}

In the study period, 75 patients were enrolled. Two patients were lost at follow-up, therefore the analysis was conducted on 73 patients. Patient and tumor characteristics are reported in Table 1. The median follow-up was of 40 months (range, 7-60 months).

The proposed fractionation regimen was well tolerated. No Grade 3 toxicity was documented. Two (2.7\%) patients experience Grade 2 erythema one month after PBI. Four (5.5\%) patients developed Grade 1 skin hyperpigmentation. Ten (13.7\%) patients had Grade 1 subcutaneous toxicity represented by a mild increased density at palpation of the irradiated tissue.

Late toxicity was assessable for all the treated patients. Two (2.7\%) patients developed Grade 2 pain 6 months after PBI. Four (5.5\%) patients developed asymptomatic fat necrosis, diagnosed at mammography. Grade 2 fibrosis was observed in 5 patients $(6.7 \%)$. No cardiovascular events were documented. Cosmetic results were judged "good/ excellent" in 69 patients (93\%), and "poor" in 6 (7\%).

No correlation was found between early and late toxicity and the type of adjuvant systemic therapy (no therapy vs. hormonal therapy vs. chemotherapy). No statistical correlation between dosimetric parameters and toxicity was found (Table 2). In particular, patients who developed Grade 2 radiation fibrosis had not higher radiation volumes to the untreated normal breast (UNB) than those without fibrosis (Table 3 ).

One patient experienced a local relapse 36 months after the end or radiotherapy, and subsequently underwent radical mastectomy. Local control rate at 5 years was $98 \%$. One patient relapse distantly 3 months after radiotherapy, corresponding to a 5-year distant metastases-free survival rate of $96.7 \%$. This patient died of disease 24 months later; the 5-year overall survival was $98.5 \%$.

\section{Discussion}

In PBI, particularly in the United States, the most commonly used fractionation scheme includes 38.5 Gy delivered in a twice-daily administration. This is based on large, robust and reproducible data, supported both from single institution experiences and from large cooperative groups
Table 1 Patient and tumor characteristics

\begin{tabular}{|c|c|}
\hline \multicolumn{2}{|l|}{ Age, years } \\
\hline median & 70 \\
\hline range & $61-85$ \\
\hline \multicolumn{2}{|l|}{ Performance Status sec. ECOG } \\
\hline $0-1$ & 66 \\
\hline $2-3$ & 7 \\
\hline \multicolumn{2}{|l|}{ Side } \\
\hline Right breast & 41 \\
\hline Left breast & 32 \\
\hline \multicolumn{2}{|l|}{ Histology } \\
\hline Invasive Ductal Carcinoma & 67 \\
\hline Invasive Lobular Carcinoma & 6 \\
\hline \multicolumn{2}{|l|}{ Tumor dimension, $\mathrm{cm}$} \\
\hline median & 1.2 \\
\hline range & $0.5-3.0$ \\
\hline \multicolumn{2}{|l|}{ T stage } \\
\hline $\mathrm{T} 1$ & 67 \\
\hline $\mathrm{T} 2$ & 6 \\
\hline \multicolumn{2}{|l|}{ N stage } \\
\hline NO & 53 \\
\hline N1mic & 8 \\
\hline $\mathrm{N} 1 \mathrm{a}$ & 2 \\
\hline Nx & 10 \\
\hline \multicolumn{2}{|l|}{ Estrogen Receptor status } \\
\hline positive & 70 \\
\hline negative & 3 \\
\hline \multicolumn{2}{|l|}{ Adjuvant therapy } \\
\hline hormonal therapy alone & 47 \\
\hline chemotherapy alone & 0 \\
\hline chemotherapy and hormonal therapy & 3 \\
\hline none & 23 \\
\hline
\end{tabular}

including the RTOG. Specifically, the RTOG 0319 was a phase II study aiming to evaluate the toxicity of threedimensional conformal PBI, delivering 10 fractions of 3.85 Gy, twice daily over 5 days [3]. With a median follow-up of 4.5 years only 2 (4\%) Grade 3 toxicities were observed. This fractionation and technique were subsequently adopted in the NSABP B39/RTOG 0413 phase III trial, comparing standard whole breast radiation therapy versus partial breast irradiation including the external beam technique [https://www.rtog.org/ClinicalTrials/ ProtocolTable/StudyDetails.aspx?study=0413], and also by other large randomized studies [https://clinicaltrials.gov/ ct2/show/NCT00282035].

The reason for adopting a one-fraction per day regimen is based on patient preference. A recent study reported 
Table 2 Dosimetric data (mean values) of patients with and without Grade 2 radiation fibrosis

\begin{tabular}{|c|c|c|c|}
\hline & $\mathrm{RF}(-)$ & $\mathrm{RF}(+)$ & $P$ value \\
\hline PTV_EVAL dimension & $119 \mathrm{ml}$ & $108 \mathrm{ml}$ & $p=$ n.s. \\
\hline PTV_EVAL/UNB ratio & $10.6 \%$ & $8.7 \%$ & $p=$ n.s. \\
\hline PTV_EVAL V95\% & $97.0 \%$ & $94.5 \%$ & $p=$ n.s. \\
\hline Mean PTV_EVAL Dose & $35.2 \mathrm{~Gy}$ & $35.2 \mathrm{~Gy}$ & $p=$ n.s. \\
\hline \multicolumn{4}{|c|}{ Heart for Left Located Tumors } \\
\hline V5\% & $5.8 \%$ & - & \\
\hline D2 cc (gy) & $2.5 \mathrm{~Gy}$ & & $p=$ n.s. \\
\hline \multicolumn{4}{|c|}{ Heart for Right Located Tumors } \\
\hline V5\% & $1.2 \%$ & $4.7 \%$ & $p=$ n.s. \\
\hline D2cc (Gy) & $1.4 \mathrm{~Gy}$ & $1.3 \mathrm{~Gy}$ & \\
\hline \multicolumn{4}{|l|}{ Ipsilateral Lung } \\
\hline V15\% & $18.3 \%$ & $17.1 \%$ & $p=$ n.s. \\
\hline V30\% & $3.5 \%$ & $4.2 \%$ & \\
\hline V60\% & $0.7 \%$ & $0.9 \%$ & \\
\hline \multicolumn{4}{|l|}{ Controlateral Lung } \\
\hline V3\% & less than $1 \%$ & less than $1 \%$ & $p=$ n.s. \\
\hline \multicolumn{4}{|l|}{ Controlateral Breast } \\
\hline V3\% & less than $1 \%$ & less than $1 \%$ & $p=$ n.s. \\
\hline \multicolumn{4}{|l|}{ Thyroid } \\
\hline V3\% & less than $1 \%$ & less than $1 \%$ & $p=n . s$. \\
\hline
\end{tabular}

Abbreviations: RF Radiation fibrosis, UNB Uninvolved normal breast, N.s. not significant

survey data describing patient's breast radiation preferences [11]. Of the 1807 women $70 \%$ preferred once-daily radiation therapy for 10 days compared with $30 \%$ who preferred the twice-daily option. Interestingly, the multivariate analysis showed that older women were more likely to prefer 10 days of once daily treatment. Moreover, a major factor influencing women's preference for whole breast irradiation over PBI was likely an aversion to twice-a-day treatment. The authors stated that they believe that their observations support the testing of novel once-daily PBI strategies in clinical trials.

We conducted a phase II prospective study to explore the toxicity profile of a novel fractionation scheme for PBI, consisting of 35 Gy delivered in 7 daily fractions. Using the linear quadratic model and the BED equation derived from this model, assuming an $\alpha / \beta$ ratio of $4 \mathrm{~Gy}$, as suggested by experiments involving irradiation of human breast cancer cell lines, this prescription would be equivalent to $55.8 \mathrm{~Gy}$ in a standard 2-Gy fractionation [12,13]. These calculations assumed that full repair takes place during the 24-h interval between fractions. In addition, because the hypofractionated regimen also represents an accelerated protocol in which the total dose is delivered in only 7 days, less tumor proliferation is expected to take place compared with that occurring during the standard treatment.
Table 3 Uninvolved normal breast dosimetric parameters (mean values) of patients with and without Grade 2 radiation fibrosis

\begin{tabular}{|c|c|c|c|}
\hline UNB & $\mathrm{RF}(-)$ & $\mathrm{RF}(+)$ & $P$ value \\
\hline v5 & $75 \%$ & $80 \%$ & $p=$ n.s. \\
\hline v10 & $69 \%$ & $74 \%$ & $p=$ n.s. \\
\hline v15 & $62 \%$ & $64 \%$ & $p=$ n.s. \\
\hline$v 20$ & $54 \%$ & $55 \%$ & $p=$ n.s. \\
\hline$v 25$ & $49 \%$ & $51 \%$ & $p=$ n.s. \\
\hline v30 & $45 \%$ & $48 \%$ & $p=$ n.s. \\
\hline v35 & $42 \%$ & $45 \%$ & $p=$ n.s. \\
\hline v40 & $39 \%$ & $41 \%$ & $p=$ n.s. \\
\hline v45 & $35 \%$ & $37 \%$ & $p=$ n.s. \\
\hline$v 50$ & $32 \%$ & $33 \%$ & $p=$ n.s. \\
\hline v55 & $29 \%$ & $30 \%$ & $p=$ n.s. \\
\hline$v 60$ & $27 \%$ & $26 \%$ & $p=$ n.s. \\
\hline v65 & $25 \%$ & $23 \%$ & $p=$ n.s. \\
\hline v70 & $23 \%$ & $21 \%$ & $p=$ n.s. \\
\hline v75 & $21 \%$ & $19 \%$ & $p=$ n.s. \\
\hline v80 & $19 \%$ & $17 \%$ & $p=$ n.s. \\
\hline v85 & $17 \%$ & $16 \%$ & $p=$ n.s. \\
\hline v90 & $15 \%$ & $14 \%$ & $p=$ n.s. \\
\hline v95 & $12 \%$ & $11 \%$ & $p=$ n.s. \\
\hline v100 & $6 \%$ & $6 \%$ & $p=$ n.s. \\
\hline v105 & $0.2 \%$ & $0.2 \%$ & $p=$ n.s. \\
\hline
\end{tabular}

Abbreviations: UNB uninvolved normal breast, $R F$ radiation fibrosis, N.s. not significant

The recent implementation of normal tissue complication probability (NTCP) model for radiation induced fibrosis after PBI supports the hypothesis that this radiation schedule would be well tolerated [7, 8]. According to the model predictions, the present fractionation scheme (35 Gy in 7 fractions) should lead to the same incidence of fibrosis as the previous one, consisting of 40 Gy in 10 daily fractions [6]. Congruent with our hypothesis, we documented that the incidence of Grade 2 fibrosis was of $6.7 \%$. The Grade 2 fibrosis reported with the previous fractionation of 40 Gy in 10 fractions was 5.9\% [6].

The low toxicity profile reported in the present study is comparable with the results of other experiences adopting daily fraction schedules for PBI. Investigators at the New York University reported the outcome of 47 patients treated in prone position with 30 Gy at 6 Gy/ fraction, delivered in 5 fractions within 10 days; with a median follow-up of 18 months they reported only late Grade 1 toxicity [14]. Researchers from the University of Florence recently published their institutional phase III randomized trial, comparing whole breast irradiation vs. external beam PBI consisting of 30 Gy in 5 daily fractions [15]. The PBI group presented significantly better 
results considering acute and late toxicity, and no Grade 2 or higher toxicities were observed in this group.

\section{Conclusions}

In conclusion, we showed that a 7 fractions schedule for PBI is safe, although a longer follow-up is needed to further ascertain late toxicity, and in particular late fibrosis. Based on the data reported in the present paper, we will continue our research in the direction of reducing the number of fractions for external beam PBI. We are now enrolling patients in a phase II trial designed to deliver $28 \mathrm{~Gy}$ in only 4 daily fractions.

\section{Abbreviations}

3D-CRT: Three dimensional conformal external beam radiotherapy; ASTRO: American society for radiation oncology; CT: Computed tomography; CTV: Clinical target volume; DRRs: Digitally reconstructed radiographs; DVH: Dose-volume histogram; IDC: Invasive ductal carcinoma; ILC: Invasive lobular carcinoma; PBI: Partial breast irradiation; PTV: Planning target volume; PTV-EVAL: Planning target volume for evaluation; RTOG: Radiation therapy oncology group; UNB: Untreated normal breast

\section{Acknowledgements}

Not applicable.

\section{Funding}

Not applicable.

\section{Availability of data and materials}

Please contact author for data requests.

\section{Authors' contributions}

MT conceived the study, collected and analyzed the patient data, and drafted the manuscript. MA carried out the dosimetric data analysis and helped to draft the manuscript. CF, TP, LM, SS, MB, EP, MM, EM participated in the pre-treatment and follow-up patients' data collection. FF, RJ and JS participated in the design of the study and helped to draft the manuscript. SM and MR participated in its design and coordination. All authors read and approved the final manuscript.

\section{Competing interests}

The authors declare that they have no competing interests.

\section{Consent for publication}

Not applicable.

\section{Ethics approval and consent to participate}

The approval from our institutional review board and written informed consent from the patients were obtained.

\section{Publisher's Note}

Springer Nature remains neutral with regard to jurisdictional claims in published maps and institutional affiliations.

\footnotetext{
Author details

${ }^{1}$ Department of Radiation Oncology, Centro di Riferimento Oncologico IRCSS, 33081 Aviano, Italy. ${ }^{2}$ Division of Medical Physics, Centro di Riferimento Oncologico IRCSS, 33081 Aviano, Italy. ${ }^{3}$ Department of Radiation Oncology, University Hospital S. Anna, Ferrara, Italy. ${ }^{4}$ Department of Pathology, Centro di Riferimento Oncologico IRCSS, 33081 Aviano, Italy. ${ }^{5}$ Department of Medical Oncology, Centro di Riferimento Oncologico IRCSS, 33081 Aviano, Italy. ${ }^{6}$ Oncology Centre, Cambridge University Hospitals NHS Foundation Trust, Cambridge, UK. ${ }^{7}$ Centro di Riferimento Oncologico IRCSS, 33081 Aviano, Italy. ${ }^{8}$ Breast Surgery Unit, Department of Oncology and Surgery, Centro di Riferimento Oncologico IRCSS, 33081 Aviano, Italy. ${ }^{9}$ Department of General Surgery, Pordenone General Hospital, 33170 Pordenone, Italy.
}

Received: 25 January 2017 Accepted: 16 May 2017

Published online: 23 May 2017

References.

1. Smith BD, Arthur DW, Buchholz TA, et al. Accelerated partial breast irradiation consensus statement from the American society for radiation oncology (ASTRO). Int J Radiat Oncol Biol Phys. 2009;74:987-1001.

2. Vicini FA, Chen $P$, Wallace $R N$, et al. Interim cosmetic results and toxicity using 3D conformal external beam radiotherapy to deliver accelerated partial breast irradiation in patients with early-stage breast cancer treated with breastconserving therapy. Int J Radiat Oncol Biol Phys. 2007:69:1124-30.

3. Vicini F, Winter K, Wong J, et al. Initial efficacy results of RTOG 0319: threedimensional conformal radiation therapy (3D-CRT) confined to the region of the lumpectomy cavity for stage I/II breast carcinoma. Int J Radiat Oncol Biol Phys. 2010;77:1120-27.

4. Jagsi R, Ben-David MA, Moran JM, et al. Unacceptable cosmesis in a protocol investigating intensity-modulated radiotherapy with active breathing control for accelerated partial-breast irradiation. Int J Radiat Oncol Biol Phys. 2010;76:71-8.

5. Hepel JT, Tokita M, MacAusland SG, et al. Toxicity of three-dimensional conformal radiotherapy for accelerated partial breast irradiation. Int J Radiat Oncol Biol Phys. 2009:75:1290-96.

6. Trovo M, Roncadin M, Polesel J, et al. Toxicity and cosmesis following partial breast irradiation consisting of 40 Gy in 10 daily fractions. Breast. 2013;22: 744-47.

7. Avanzo M, Stancanello J, Trovo M, et al. Complication probability model for subcutaneous fibrosis based on published data of partial and whole breast irradiation. Phys Med. 2012;28:296-306.

8. Avanzo M, Trovo M, Stancanello J, et al. Hypofractionation of partial breast irradiation using radiobiological models. Phys Med. 2015;31:1022-28.

9. Trovo M, Polesel J, Biasutti C, et al. Fiducial markers for image-guided partial breast irradiation. Radiol Med. 2013;118:1212-19.

10. Trovo M, Sartor G, Chiovati $P$, et al. Forward intensity modulated radiation therapy for partial breast irradiation confers high dose homogeneity and conformation to the target volume. Int J Radiat Oncol Biol Phys. 2010;78:S747.

11. Hoops DJ, Kaziska D, Chapin P, et al. Patient preferences and physician practice patterns regarding breast radiotherapy. Int J Radiat Oncol Biol Phys. 2012;82:674-81.

12. Barendsen GW. Dose fractionation, dose rate and iso-effect relationships for normal tissue responses. Int J Radiat Oncol Biol Phys. 1982;8:1981-87.

13. Steel G, Deacon J, Duchesne GM, et al. The dose-rate effect in human tumour cells. Radiother Oncol. 1987:9:299-310.

14. Formenti SC, Truong MT, Godberg JD, et al. Prone accelerated partial breast irradiation after breast-conserving surgery: preliminary clinical results and dosevolume histogram analysis. Int J Radiat Oncol Biol Phys. 2004;60:493-504.

15. Livi L, Meattini I, Marrazzo L, et al. Accelerated partial breast irradiation using intensity-modulated radiotherapy versus whole breast irradiation: 5-year survival analysis of a phase 3 randomised controlled trial. Eur J Cancer. 2015; 51:451-63.
Submit your next manuscript to BioMed Central and we will help you at every step:

- We accept pre-submission inquiries

- Our selector tool helps you to find the most relevant journal

- We provide round the clock customer support

- Convenient online submission

- Thorough peer review

- Inclusion in PubMed and all major indexing services

- Maximum visibility for your research

Submit your manuscript at www.biomedcentral.com/submit
) Biomed Central 